\title{
A safe protocol to identify low-risk patients with COVID-19 pneumonia for outpatient management
}

\author{
Francisco Javier Teigell Muñoz ${ }^{1,2}\left(\right.$ Elena García-Guijarro ${ }^{1,2} \cdot$ Paula García-Domingo ${ }^{1,2} \cdot$ Guadalupe Pérez-Nieto $^{1,2}$. \\ Fernando Roque Rojas ${ }^{1,2}$ - María García-Peña ${ }^{3}$ - María Antonia Nieto Gallo ${ }^{3}$ José Antonio Melero Bermejo ${ }^{1,2}$. \\ María Teresa de Guzman García-Monge ${ }^{1,2}$. Juan José Granizo ${ }^{4,5}$
}

Received: 3 November 2020 / Accepted: 1 February 2021 / Published online: 23 February 2021

(c) Società Italiana di Medicina Interna (SIMI) 2021

\begin{abstract}
The coronavirus disease 2019 (COVID-19) outbreak has made it necessary to rationalize health-care resources, but there is little published data at this moment regarding ambulatory management of patients with COVID-19 pneumonia. The objective of the study is to evaluate the performance of a protocol for ambulatory management of patients with COVID-19 pneumonia regarding readmissions, admission into the Intensive Care Unit (ICU) and deaths. Also, to identify unfavorable prognostic factors that increase the risk of readmission. This is a prospective cohort study of patients with COVID-19 pneumonia discharged from the emergency ward of Infanta Cristina Hospital (Madrid, Spain) that met the criteria of the hospital protocol for outpatient management. We describe outcomes of those patients and compare those who needed readmission versus those who did not. We use logistic regression to explore factors associated with readmissions. A total of 314 patients were included, of which 20 (6.4\%) needed readmission, and none needed ICU admission nor died. At least one comorbidity was present in $29.9 \%$ of patients. Hypertension, leukopenia, lymphocytopenia, increased lactate dehydrogenase $(\mathrm{LDH})$ and increased aminotransferases were all associated with a higher risk of readmission. A clinical course of 10 days or longer, and an absolute eosinophil count over $200 / \mu \mathrm{L}$ were associated with a lower risk. After the multivariate analysis, only hypertension (OR 4.99, CI 1.54-16.02), temperature over $38^{\circ} \mathrm{C}$ in the emergency ward (OR 9.03, CI 1.89-45.77), leukopenia (OR 4.92, CI 1.42-17.11) and increased LDH (OR 6.62, CI 2.82-19.26) remained significantly associated with readmission. Outpatient management of patients with low-risk COVID-19 pneumonia is safe, if adequately selected. The protocol presented here has allowed avoiding 30\% of the admissions for COVID-19 pneumonia in our hospital, with a very low readmission rate and no mortality.
\end{abstract}

Keywords COVID-19 · Outpatient management $\cdot$ COVID-19 pneumonia $\cdot$ SARS-CoV-2 $\cdot$ Low risk COVID-19 pneumonia

Francisco Javier Teigell Muñoz javier.teigell@gmail.com

1 Department of Emergency Medicine and Internal Medicine, Infanta Cristina University Hospital, Madrid, Spain

2 Department of Medicine, Complutense University, Madrid, Spain

3 Department of Pathology, Infanta Cristina University Hospital, Madrid, Spain

4 Preventive Medicine Unit, Infanta Cristina University Hospital, Madrid, Spain

5 Institute of Investigations Puerta de Hierro-Segovia de Arana, Madrid, Spain

\section{Introduction}

Spain has been one of the countries most severely hit by the first wave of worldwide SARS-CoV-2 pandemic, with over 230,000 confirmed cases by May 31st, 2020, and approximately 28,000 deaths [1]. Although most patients only presented with mild episodes, many developed pneumonia, some of them developed severe respiratory failure and eventually, death. As it was especially difficult to identify the patients at higher risk of unfavorable evolution, the initial clinical strategy was to admit all patients diagnosed with COVID-19 and pneumonia for treatment and close monitoring.

However, the great accumulated incidence of pneumonia cases in a short time span, showing an exponential growth of 
the amount of hospital admissions, led not only to a shortening in material and human resources but also to a high risk of collapse for the health system, which could itself lead to a decrease in the quality of patient care and hence to an increase in mortality.

It became necessary to create a strategy to rationalize health-care resources and prioritize inpatient attention to those in greater need. Within the Section of Emergency Medicine and Internal Medicine at the Infanta Cristina Hospital (ICH) in Madrid, Spain, we developed a protocol to select those patients which would truly benefit from inpatient care and those who could be cared for via outpatient telephonic follow-up, based on established criteria.

In this study, we describe our protocol for the ambulatory care for patients with low-risk COVID-19 pneumonia and evaluate its performance during the first wave of the current pandemic.

\section{Methods}

\section{Study design and sample}

A cohort of patients discharged between March 17th and April 25th, 2020, from the emergency ward of the ICH with clinical and radiological diagnoses of COVID-19 pneumonia was prospectively evaluated.

Among all patients managed as outpatients, only those who met the discharge criteria established in the hospital protocol were included. These criteria (Table 1) were elaborated after reviewing the literature available at the moment, emphasizing the work of and Fei Zhou et al. [2], and
Chaomin Wu et al.[3], where they describe both favorable and unfavorable prognostic factors in COVID-19 patients. According to the protocol, a chest radiograph was performed on all patients with fever and/or other symptoms suggesting COVID-19. Those with no pathological findings in the radiograph were discharged whenever their clinical status allowed for it. In radiologically dubious cases, chest CT or lung ultrasound were performed. The protocol also recommended performing a simplified walking test to assess lung capacity. The test was conducted by having the patient walk fast $30 \mathrm{~m}$, and was considered positive whenever oxygen saturation, as measured by pulse oximetry, decreased by 5 percent or more. RT-PCR for SARS-CoV-2 was performed via nasopharyngeal swab, according to the instructions from the official health authority at the time of each evaluation. Patients with suspected COVID-19 pneumonia could be discharged whenever they did not meet major admission criteria (Table 1). Admission was also encouraged in cases with more than five minor criteria. All patients were discharged with home therapy, according to the protocol of the hospital valid at the time of discharge.

The telephone follow-up started the day after discharge, which could be daily or once every other day, according to the patient's status. In each call, a basic questionnaire was performed, which included questions about the presence of fever, dyspnea, cough, chest pain, digestive symptoms and drug tolerability. Patients with non-relenting fever despite medication, fever persisting longer than 3 days after the start of follow-up, resting or minimal-exertion dyspnea, inability to feed or limiting asthenia, were referred back to the emergency ward for on-site evaluation, complementary workup or, eventually,
Table 1 Discharge criteria according to the protocol of the Infanta Cristina University Hospital
Infanta Cristina Hospital's discharge criteria (no major criteria should be met, $\leq 5$ minor criteria are allowed)

\begin{tabular}{|c|c|}
\hline Major criteria & Minor criteria \\
\hline Age $>70$ years & Age $50-70$ years \\
\hline $\mathrm{SaO} 2<95 \%$ and/or positive walking-test & $\begin{array}{l}\text { Comorbidities }^{\mathrm{a}} \text { ( } 1 \text { point each, maximum allowed } \\
3 \text {, if non-severe) }\end{array}$ \\
\hline Bronchospasm & Aminotransferases $>2 \times$ the upper limit of normal ${ }^{b}$ \\
\hline Radiological affection $>50 \%^{\mathrm{c}}$ & Increased LDH above upper limit of normal ${ }^{b}$ \\
\hline $\mathrm{CPR}>100 \mathrm{mg} / \mathrm{L}$ & CPR $50-100 \mathrm{mg} / \mathrm{L}$ \\
\hline $\mathrm{D}$-dimer $>1,000 \mathrm{ng} / \mathrm{mL}$ & D-dimer $500-1,000 \mathrm{ng} / \mathrm{mL}$ \\
\hline Lymphocytes $<800 / \mu \mathrm{L}$ & Lymphocytes $800-1,200 / \mu \mathrm{L}$ \\
\hline CURB score $\geq 1$ & \\
\hline
\end{tabular}

$\mathrm{SaO} 2$ baseline oxygen saturation, $L D H$ lactate-dehydrogenase, $C P R$ C-reactive protein, $C U R B$ confusion, urea, respiratory rate, low blood pressure

${ }^{a}$ Comorbidities: persistent asthma, chronic obstructive pulmonary disease, obstructive sleep apnea-hypopnea syndrome, ischemic heart disease, active cancer, kidney failure, liver failure, hypertension, diabetes mellitus, obesity, immunosuppression

${ }^{\mathrm{b}}$ Upper limit of normal: AST $<40 \mathrm{U} / \mathrm{L} ; \mathrm{ALT}<50 \mathrm{U} / \mathrm{L} ; \mathrm{LDH}<250 \mathrm{U} / \mathrm{L}$

${ }^{\mathrm{c}}$ Interpreted by treating physician team 
inpatient admission. During the study period, some digital pulse oximeters became available, and they were lent to the patients at highest risk, which allowed for a more adequate surveillance of their respiratory status.

Those patients that did not meet the protocol criteria but were also discharged (e.g. those meeting major admission criteria) were excluded from analysis, as were those for which the telephone follow-up was deemed unfeasible because of mental illness, language barrier, etc. Pregnant patients have also been excluded, as they were followed by the Section of Gynecology and Obstetrics of our institution. Patients with intermediate to low clinical suspicion and negative RT-PCR were also excluded (Fig. 1).

\section{Study objectives}

The primary objective was to evaluate the performance of the protocol for ambulatory management according to the proportion of readmissions due to complications or worsening, admissions into the Intensive Care Unit (ICU) and deaths. The secondary objective was to identify unfavorable prognostic factors that increase the risk of readmission, ICU admission and/or death.

\section{Statistical analysis}

IBM SPSS 16.0 was used for analysis. Quantitative variables are displayed as median, interquartile range (IQR) and eventually range; categorical variables are displayed as absolute and relative (\%) frequency. For comparative analysis, both the $\chi^{2}$ and Fisher's exact test were used in the case of categorical variables, and Mann-Whitney's test for non-categorical quantitative variables. To explore the risk factors associated with readmission, both univariate and multivariate logistic regression models were used. After univariate analysis, considering the low rate of readmission (20 in total), only 4 variables were used in the multivariate analysis. Only those differences with an associated bilateral alpha error lower than 0.05 were considered significant.

\section{Results}

Clinical and demographical features of the sample are shown in Table 2. Laboratory tests are available for all but one patient (Table 3). The statistical distribution of radiological pulmonary abnormalities is also shown in Table 3 . Three patients had a normal chest radiograph, and the diagnosis of pneumonia was made via lung ultrasound or chest tomography. RT-PCR for SARS-CoV-2 was performed on 138
Fig. 1 Population included in the analysis

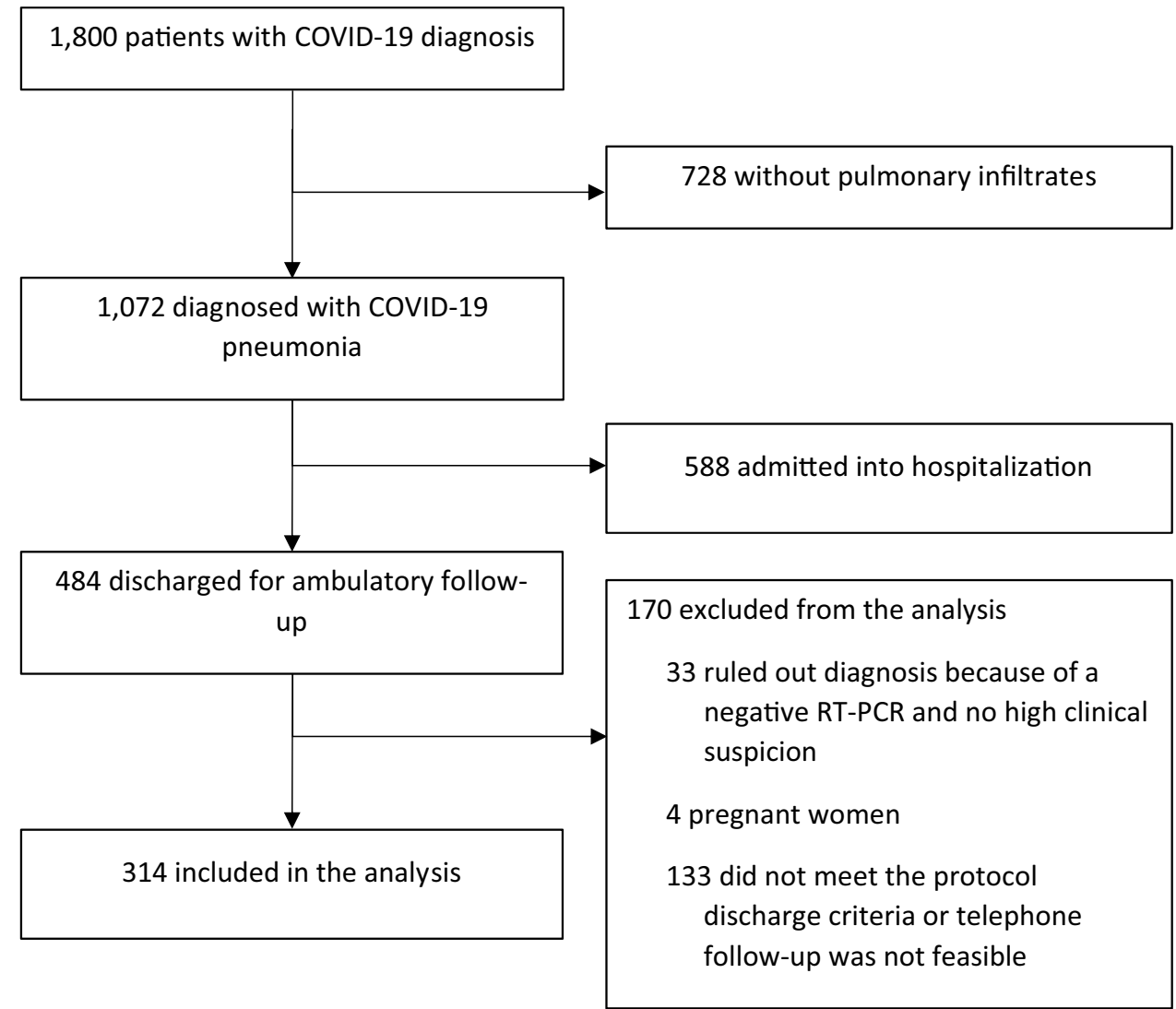


Table 2 Demographic and clinical features

\begin{tabular}{|c|c|c|c|c|}
\hline & $\begin{array}{l}\text { Total } \\
n=314\end{array}$ & $\begin{array}{l}\text { Readmission } \\
n=20\end{array}$ & $\begin{array}{l}\text { No readmission } \\
n=294\end{array}$ & $p$ value \\
\hline Age (years) & $45.0(40.0-53.0)$ & $46.5(42.3-50.8)$ & $45(39-53)$ & 0.501 \\
\hline $50-70$ & $107(34.1 \%)$ & $6(30 \%)$ & $101(34.4 \%)$ & 0.69 \\
\hline \multicolumn{5}{|l|}{ Sex } \\
\hline Female & $165(52.5 \%)$ & $8(40 \%)$ & $157(53.4 \%)$ & 0.24 \\
\hline Male & $149(47.5 \%)$ & $12(60 \%)$ & $137(46.6 \%)$ & - \\
\hline Active smoking & $21 / 124(6.7 \%)$ & $1 / 9(11.1 \%)$ & 20/115 (17.4\%) & 0.38 \\
\hline \multicolumn{5}{|l|}{ Comorbidities } \\
\hline Number of comorbidities per patient & $0(0-1)$ & $0(0-1)$ & $0(0-1)$ & 0.369 \\
\hline Presence of at least one comorbidity & $94(29.9 \%)$ & $8(40 \%)$ & $86(29.3 \%)$ & 0.144 \\
\hline Persistent asthma & $15(4.8 \%)$ & $0(0 \%)$ & $15(5.1 \%)$ & 0.36 \\
\hline COPD & $1(0.3 \%)$ & $0(0 \%)$ & $1(0.3 \%)$ & - \\
\hline OSAHS & $4(1.3 \%)$ & $0(0 \%)$ & $4(1.3 \%)$ & - \\
\hline Ischemic heart disease & $5(1.6 \%)$ & $1(5 \%)$ & $4(1.4 \%)$ & 0.282 \\
\hline Active cancer & $1(0.3 \%)$ & $0(0 \%)$ & $1(0.3 \%)$ & - \\
\hline Kidney/liver failure & $0(0 \%)$ & - & - & - \\
\hline Hypertension & $45(14.3 \%)$ & $6(30 \%)$ & $39(13.3 \%)$ & 0.039 \\
\hline Use of ACE-inhibitors or ARBs & $35(11.2 \%)$ & $4(20 \%)$ & $31(10.5 \%)$ & 0.26 \\
\hline Diabetes mellitus & $17(5.4 \%)$ & $3(15 \%)$ & $14(4.8 \%)$ & 0.085 \\
\hline Obesity & $17(5.4 \%)$ & $3(15 \%)$ & $14(4.8 \%)$ & 0.085 \\
\hline Use of immune suppresants & $10(3.2 \%)$ & $0(0 \%)$ & $10(3.4 \%)$ & 0.512 \\
\hline Time since symptom start & $7.0(5-12)$ & $7(4.3-7)$ & $8(5-12)$ & 0.009 \\
\hline$<10$ days & $193(62.3 \%)$ & $20(100 \%)$ & $177(59.7 \%)$ & - \\
\hline$\geq 10$ days & $117(37.7 \%)$ & $0(0)$ & $117(40.3 \%)$ & $<0.001$ \\
\hline Fever (referred by the patient) & $238(75.8 \%)$ & $18(90 \%)$ & $220(75 \%)$ & 0.17 \\
\hline Cough & $246(78.3 \%)$ & $16(80 \%)$ & $230(78.5 \%)$ & 0.87 \\
\hline Dyspnea & $190(60.5 \%)$ & $12(60 \%)$ & $178(60.8 \%)$ & 0.95 \\
\hline Diarrhea or vomiting & $100(31.8 \%)$ & $6(30 \%)$ & $94(32.2 \%)$ & 0.84 \\
\hline Arterial oxygen saturation in the emergency ward & $98(96-98)$ & $97.1(96-98)$ & $97.6(96-98)$ & 0.18 \\
\hline Heart rate in the emergency ward (bpm) & $89(80-200)$ & $90(85-104)$ & $91.5(80-99)$ & 0.565 \\
\hline Temperature in the emergency ward $\left({ }^{\circ} \mathrm{C}\right)$ & $36.6(36.4-36.9)$ & $36.6(36.4-37.1)$ & $36.6(36.4-36.9)$ & 0.634 \\
\hline$\geq 38^{\circ} \mathrm{C}$ & $11(3.5 \%)$ & $4(20 \%)$ & $7(2.4 \%)$ & 0.003 \\
\hline Abnormal lung auscultation & $91(29 \%)$ & $8(40 \%)$ & $83(28.3 \%)$ & 0.27 \\
\hline
\end{tabular}

$C O P D$ chronic obstructive pulmonary disease, $O S H A S$ obstructive sleep apnea-hypopnea syndrome, $A C E$ angiotensin-converting enzyme, $A R B$ angiotensin receptor blockers

(43.9\%) patients; 93 were positive $(67.4 \%$ of the performed PCR tests), 40 (29.0\%) were negative and 5 (3.6\%) gave indeterminate results. The patients included in the ambulatory management program had a median of one minor criterion (IQR 0-2). $74.5 \%$ of them had one or more minor criteria, and $9.6 \%$ had more than three.

Table 4 shows the therapy prescribed upon discharge. It shows also the data of the follow-up and patient course. The telephone follow-up period had a median of 5 days starting from the day of discharge (IQR 4-6, range 1-11), with a median of 3 telephone calls per patient (IQR 3-4, range 1-10). Depending on the availability in each moment, in the end, $15.6 \%$ of patients were given a portable pulse-oximeter to facilitate their monitoring. 62 patients $(19.7 \%)$ were reevaluated in the emergency ward at least one second time. Half of them $(9.9 \%)$ were referred because of medical criteria (almost all of them because of persistent fever or dyspnea), whereas the other half $(9.9 \%)$ presented by their own choice. 42 of those patients were newly discharged, so 20 patients $(6.4 \%)$ ultimately needed inpatient hospital admission. The most common reason for readmission $(50 \%)$ was respiratory worsening with the development of hypoxemia (SatO2<95\%), with one only case of respiratory failure (SatO2 88\%). One patient was readmitted with a diagnosis of segmental pulmonary 
Table 3 Laboratory, imaging and microbiology test results

\begin{tabular}{|c|c|c|c|c|}
\hline & $\begin{array}{l}\text { Total } \\
n=313\end{array}$ & $\begin{array}{l}\text { Readmission } \\
n=19\end{array}$ & $\begin{array}{l}\text { No readmission } \\
n=294\end{array}$ & $p$ value \\
\hline \multicolumn{5}{|l|}{ Laboratory findings } \\
\hline Leukocytes $(/ \mu \mathrm{L})$ & $6390(5132-7960)$ & $4920(3960-6300)$ & $6520(5220-8110)$ & 0.001 \\
\hline$<4000$ & $27(8.6 \%)$ & $5(26.3 \%)$ & $22(7.4 \%)$ & 0.004 \\
\hline Lymphocytes $(/ \mu \mathrm{L})$ & $1599(1100-2000)$ & $1200(1093-1700)$ & $1600(1200-2000)$ & 0.041 \\
\hline $800-1200$ & $80(25.5 \%)$ & $9(47.4 \%)$ & $71(24.1 \%)$ & 0.025 \\
\hline$\geq 1200$ & $233(74.5 \%)$ & $10(52.6 \%)$ & $223(75.9 \%)$ & \\
\hline Eosinophils $(/ \mu \mathrm{L})$ & $100(0-100)$ & $0(0-0)$ & $100(0-100)$ & $<0.001$ \\
\hline$>200$ & $28 / 301(9.3 \%)$ & $0(0 \%)$ & $28 / 282(9.9 \%)$ & 0.234 \\
\hline Platelets $\left(\times 10^{3} / \mu \mathrm{L}\right)$ & $226(186-283)$ & $163(133-189)$ & $231(190-286)$ & $<0.001$ \\
\hline Lactate-dehydrogenase (U/L) & $197(166-235)$ & $253(234-287)$ & $193(164-228)$ & $<0.001$ \\
\hline$>250$ & $46 / 270(16.5 \%)$ & $9 / 18(50 \%)$ & $37 / 261(14.2 \%)$ & $<0.001$ \\
\hline Creatinine (mg/dL) & $0.83(0.7-0.98)$ & $0.98(0.81-1.15)$ & $0.82(0.70-0.97)$ & 0.005 \\
\hline Aspartate-aminotransferase (U/L) & $29(21-37.25)$ & $38(33-48)$ & $28(21-37)$ & 0.004 \\
\hline Alanine-aminotransferase (U/L) & $38(28-55)$ & $46(36.5-60.5)$ & $38(28-55)$ & 0.299 \\
\hline Abnormal aminotransferases ${ }^{\mathrm{b}}$ & $121(38.5 \%)$ & $12(60 \%)$ & $109(37.1 \%)$ & 0.042 \\
\hline C-reactive protein (mg/L) & $18.6(4.5-35)$ & $29.3(17.3-29.3)$ & $16.8(4.3-34.3)$ & 0.012 \\
\hline$<50$ & $264(84.4 \%)$ & $14(73.7 \%)$ & $250(84.5 \%)$ & 0.207 \\
\hline $50-100$ & $49(15.6 \%)$ & $5(26.3 \%)$ & $44(15.5 \%)$ & \\
\hline Prothrombin activity (\%) & $92(84-98)$ & $98(89-102)$ & $91(84-98)$ & 0.175 \\
\hline D-dimer (ng/mL) & $360(250-530)$ & $365(267-435)$ & $360(250-530)$ & 0.901 \\
\hline$<500$ & $226(72.2 \%)$ & $15(79 \%)$ & $211(71.6 \%)$ & 0.569 \\
\hline $500-1000$ & $87(27.8 \%)$ & $4(21 \%)$ & $83(28.4 \%)$ & \\
\hline \multicolumn{5}{|l|}{ Minor criteria } \\
\hline Number of minor criteria & $1(0-2)$ & $2(1-3)$ & $1(0-2)$ & 0.026 \\
\hline Minor criteria $\geq 1$ & $234(74,5 \%)$ & $19(95 \%)$ & $215(73,1 \%)$ & 0.001 \\
\hline Radiological findings & & $n=20$ & $n=294$ & 0.223 \\
\hline Normal radiograph & $3(1 \%)$ & $3(1 \%)$ & $0(0 \%)$ & \\
\hline Unilobar affection & $105(33.4 \%)$ & $4(20 \%)$ & $101(34.4 \%)$ & 0.188 \\
\hline Unilateral multilobar affection & $33(10.5 \%)$ & $5(25 \%)$ & $28(9.5 \%)$ & \\
\hline Bilateral affection $<50 \%$ & $172(54.8 \%)$ & $11(55 \%)$ & $161(54.8 \%)$ & \\
\hline Virological diagnosis & & $n=20$ & $n=294$ & \\
\hline RT-PCR SARS-CoV-2 performed & $138 / 314(43.9 \%)$ & $15(75 \%)$ & $123(41.8 \%)$ & 0.004 \\
\hline Positive (\% of performed) & $93(67.4 \%)$ & $14(93.3 \%)$ & $79(64 \%)$ & 0.075 \\
\hline Negative (\% of performed) & $40(29 \%)$ & $1(5 \%)$ & $39(31.7 \%)$ & \\
\hline Inhibited or indeterminate ( $\%$ of performed) & $5(3.6 \%)$ & $0(0 \%)$ & $5(4.1 \%)$ & \\
\hline RT-PCR SARS-CoV-2 not performed & $176(56.1 \%)$ & $5(25 \%)$ & $171(58,2 \%)$ & 0.004 \\
\hline
\end{tabular}

${ }^{\mathrm{a}}$ In those cases, the diagnosis was made via chest $\mathrm{CT}$ or lung ultrasound

${ }^{\mathrm{b}} \mathrm{AST}$ and/or ALT above upper limit of normal: AST $<40 \mathrm{U} / \mathrm{L} ;$ ALT $<50 \mathrm{U} / \mathrm{L}$

embolism. $60 \%$ of readmissions presented again by their own choice. All patients that were readmitted did so within the first 6 days of follow-up (median 3, IQR 2-4, range 1-6). During admission, three patients developed severe respiratory failure (defined by oxygen requirements above $6 \mathrm{~L} / \mathrm{min}$, need for high flow oxygen, or noninvasive mechanical ventilation). No patients needed ICU admission or died.

\section{Secondary objectives}

The patients requiring readmission were compared with the ones that did not, to detect significant differences. As shown in Table 2, there were no differences regarding age or sex. Among the patients that required readmission, there was a higher proportion of hypertension $(30.0 \%$ vs. $13.3 \%$, $p 0.039$ ). The frequency of diabetes and obesity was also higher but did not reach statistical significance (15\% vs. 
Table 4 Prescribed treatment, follow-up and evolution

\begin{tabular}{|c|c|c|c|c|}
\hline & $\begin{array}{l}\text { Total } \\
n=314\end{array}$ & $\begin{array}{l}\text { Readmission } \\
n=20\end{array}$ & $\begin{array}{l}\text { No readmission } \\
n=294\end{array}$ & $p$ value \\
\hline \multicolumn{5}{|l|}{ Prescribed treatment } \\
\hline Hydroxychloroquine or chloroquine & $304(96.8 \%)$ & $20(100 \%)$ & $284(96.6 \%)$ & 0.51 \\
\hline Beta-lactams & $107(34.1 \%)$ & $11(55.0 \%)$ & $96(32.8 \%)$ & 0.043 \\
\hline Azithromycin & $266(84.7 \%)$ & $11(55.0 \%)$ & $255(86.7 \%)$ & $<0.001$ \\
\hline Lopinavir/ritonavir & $13(4.1 \%)$ & $0(0 \%)$ & $13(4.4 \%)$ & 0.41 \\
\hline Non-steroid antiinflammatory drugs & $89(28.3 \%)$ & $7(35.0 \%)$ & $82(28.2 \%)$ & 0.514 \\
\hline \multicolumn{5}{|l|}{ Follow-up and evolution } \\
\hline Follow-up days per patient (until end or follow-u or readmission) & $5(4-6)$ & $3(2-4)$ & $5(4-6)$ & $<0.001$ \\
\hline Number of telephone calls per patient & $3(3-4)$ & $3(1-3)$ & $3(3-4)$ & 0.004 \\
\hline Use of pulse oximeter during follow-up & $49(15.6 \%)$ & $0(0 \%)$ & $49(16.7 \%)$ & 0.053 \\
\hline Patients reevaluated in the emergency ward & $62(19.7 \%)$ & $20(100 \%)$ & $42(14.3 \%)$ & $<0.001$ \\
\hline Referred by a physician & $31(9.9 \%)$ & $8(40 \%)$ & $23(7.8 \%)$ & $<0.001$ \\
\hline By patient's own choice & $31(9.9 \%)$ & $12(60 \%)$ & $19(6.5 \%)$ & $<0.001$ \\
\hline Need for inpatient admission & $20(6.4 \%)$ & - & - & - \\
\hline $\mathrm{SaO} 2$ in the emergency ward at the time of readmission $(\%)$ & - & $94(92.7-96)$ & - & - \\
\hline $\mathrm{SaO} 2<90 \%$ at the time of admission & - & $1(5.0 \%)$ & - & - \\
\hline $\mathrm{SaO} 290-94 \%$ at the time of admission & - & $9(45.0 \%)$ & - & - \\
\hline Severe respiratory failure ${ }^{\mathrm{a}}$ during inpatient admission & $3(1 \%)$ & $3(15.0 \%)$ & - & - \\
\hline Intensive care unit admission & $0(0 \%)$ & $0(0 \%)$ & - & - \\
\hline Death & $0(0 \%)$ & $0(0 \%)$ & $0(0 \%)$ & - \\
\hline
\end{tabular}

${ }^{a}$ Severe respiratory failure was defined as oxygen requirements $>6 \mathrm{Lpm}$, need for high flow oxygen or noninvasive mechanical ventilation

$4.8 \%$ in both cases, $p$ 0.085). There were no other differences regarding comorbidities, nor in the total number of comorbidities per patient. Referred symptoms were similarly distributed in both groups, but a temperature equal or higher $38^{\circ} \mathrm{C}$ measured within the emergency ward was more frequent among the patients that did require readmission $(20.0 \%$ vs. $2.4 \%, p$ 0.003). The readmitted patients showed a significantly shorter time period since the initial symptoms (7.0 vs. 8.0 days; $p$ 0.009). Not one patient with 10 or more days of disease progression at the time of presentation was readmitted.

The patients that required readmission showed significantly lower total white blood cell, lymphocyte, eosinophil and platelet counts, and subsequently a higher rate of leukopenia, lymphocytopenia and thrombocytopenia (Table 3). There were no readmissions among the 28 patients with more than 200 eosinophils per microliter. Readmitted patients had higher levels of $\mathrm{LDH}, \mathrm{C}$ reactive protein, creatinine and liver enzymes. There were no differences in D-dimer or prothrombin activity. Readmitted patients also had significantly more minor criteria.

No significant differences were detected in radiological patterns, although a lower rate of unilobar pneumonia was observed in the patients that were readmitted (Table 3). There was also a higher proportion of patients with positive RT-PCR in that group, but it did not reach statistical signification.

The readmitted patients received beta-lactams significantly more ( 55.0 vs. $32.8 \%, p 0.043$ ) and azithromycin significantly less $(55.0$ vs. $86.7 \%, \mathrm{p}<0.001)$. There were no differences between groups regarding hydroxychloroquine or chloroquine, lopinavir/ritonavir or NSAIDs.

In the univariate analysis, hypertension, leukopenia, lymphocytopenia, increased LDH, increased aminotransferases and use of beta-lactams were associated with a higher risk of readmission (Table 5). On the contrary, a clinical course of 10 days or longer, an absolute eosinophil count over 200/ $\mu \mathrm{L}$ and use of azithromycin were associated with a lower risk. However, after multivariate analysis, only hypertension (OR 4.99, CI 1.54-16.02), temperature over $38^{\circ} \mathrm{C}$ in the emergency ward (OR 9.03, CI 1.89-45.77), leukopenia (OR 4.92, CI 1.42-17.11) and increased LDH (OR 6.62, CI $2.82-19.26)$ remained significantly associated.

\section{Discussion}

This study describes the experience of applying a management protocol within an emergency Ward to patients with COVID-19, identifying those patients presenting mild 
Table 5 Risk factors associated with inpatient readmission

\begin{tabular}{|c|c|c|c|c|c|c|}
\hline & Univariate OR & CI $95 \%$ & $p$ value & Multivariate OR & CI $95 \%$ & $p$ value \\
\hline Age (years) & 1.01 & $(0.965-1.056)$ & 0.501 & & & \\
\hline Sex (female vs. male) & 0.582 & $(0.23-1.47)$ & 0.25 & & & \\
\hline Comorbidities (present vs. absent) & 1.982 & $(0.78-5.04)$ & 0.144 & & & \\
\hline Hypertension (yes/no) & 2.802 & $(1.02-7.63)$ & 0.039 & 4.99 & $(1.54-16.02)$ & 0.007 \\
\hline Obesity (yes/no) & 3.529 & $(0.93-13.47)$ & 0.085 & & & \\
\hline Diabetes mellitus (yes/no) & 3.529 & $(0.93-13.47)$ & 0.085 & & & \\
\hline Use of ACE-inhibitors or ARBs (yes/no) & 2.121 & $(0.667-6.75)$ & 0.26 & & & \\
\hline Time since symptom start ( $\geq 10$ vs. $<10)$, days & 0.896 & $(0.85-0.94)$ & $<0.001$ & & & \\
\hline Radiological affection (unilobar vs. others) & 0.478 & $(0.16-1.47)$ & 0.188 & & & \\
\hline Temperature in the emergency ward ( $\geq 38$ vs. $<38$ ), ${ }^{\circ} \mathrm{C}$ & 10.25 & $(2.71-38.66)$ & 0.003 & 9.03 & $(1.89-45.77)$ & 0.006 \\
\hline Leukocytes $(<4000$ vs $>4000), / \mu \mathrm{L}$ & 4.49 & $(1.47-13.67)$ & 0.004 & 4.92 & $(1.42-17.11)$ & 0.012 \\
\hline Lymphocytes (800-1200 vs > 1200), / $\mu \mathrm{L}$ & 2.817 & $(1.1-7.22)$ & 0.025 & & & \\
\hline $\mathrm{LDH}(>250$ vs $<250), \mathrm{U} / \mathrm{L}$ & 6.054 & $(2.26-16.25)$ & $<0.001$ & 6.63 & $(2.82-19.26)$ & 0.001 \\
\hline Eosinophils (>200 vs $\leq 200), / \mu \mathrm{L}$ & 0.93 & $(0.90-0.96)$ & 0.234 & & & \\
\hline C-reactive protein $(50-100$ vs $<50), \mathrm{mg} / \mathrm{L}$ & 1.948 & $(0.67-5.68)$ & 0.012 & & & \\
\hline Aminotransferases (abnormal vs. normal) & 2.546 & $(1.01-6.42)$ & 0.042 & & & \\
\hline D-dimer $(500-1000$ vs $<500), \mathrm{ng} / \mathrm{mL}$ & 0.583 & $(0.16-2.10)$ & 0.569 & & & \\
\hline Azithromycin & 0.187 & $(0.07-0.48)$ & $<0.001$ & & & \\
\hline Beta-lactams & 2.508 & $(1.01-6.26)$ & 0.043 & & & \\
\hline Non-steroid antiinflammatory drugs & 1.37 & $(0.53-3.56)$ & 0.514 & & & \\
\hline
\end{tabular}

$A C E$ angiotensin-converting enzyme, $A R B$ angiotensin receptor blockers

cases and a low risk for unfavorable evolution, performing outpatient treatment and follow-up, and avoiding their admission as inpatients. The discharge criteria gathered in this protocol were based on the risk factors for unfavorable development that were described in the available literature $[2,3]$, which have been described for hospitalized patients but lack validation for use on outpatients.

The results of our study show that the ambulatory management of patients with low-risk COVID-19 pneumonia is safe, by showing a very low rate of readmissions and no associated mortality. As a reference, both the readmission $(6.4 \%)$ and mortality ( $0 \%)$ rates of our 314-patient cohort is akin to the FINE I (5.1\% readmissions, 0\% mortality) and II ( $8.2 \%$ and $0.4 \%)$ categories used for communityacquired pneumonia, as published in 1997 by Fine et al. [4] on a cohort of 587 and 244 patients, respectively. Currently, there are no therapies available with proven efficacy against mild COVID-19, and hence, it is likely that the good prognosis of our patients is more closely related to having been adequately identified than to the therapy administered. Our study shows that it is possible to manage patients with some comorbidities such as hypertension, DM, obesity or asthma as outpatients, whenever such patients are in a good clinical condition. However, the frequency of other conditions (chronic obstructive pulmonary disease, ischemic heart disease, kidney disease or chronic liver disease) in our cohort has been very low, so we are not able to surely say that our results are applicable to such patients.

Second, applying a protocol like the one proposed allows avoiding a sizable amount of inpatient admissions for COVID-19 pneumonia (almost 30\% in our experience). The lower pressure on the health-care system due to this lower amount of admissions leaves more resources, both material and human, available in the emergency and hospitalization wards. Those newly available resources for the care of severely ill patients could account for a benefit in their clinical development.

Third, this study allows us to identify the prognostic factors for both good and bad evolution in outpatients with COVID-19 pneumonia. Some of these factors have been previously described for hospitalized patients. Such is the case for hypertension, leukopenia, lymphocytopenia, renal function impairment or LDH and liver enzyme elevations. Some studies like the one done by Liu et al. [5], or Sun et al. [6] hint at the fact that a high eosinophil count might be a positive prognostic factor. On that note, the patients in our sample that were not readmitted showed a greater eosinophil median, and no patient with an eosinophil count over 200/ $\mu \mathrm{L}$ was readmitted. Mateos et al. have recently described the prognostic value of eosinophil recovery in COVID-19 [7]. Even though the physiological explanation remains unclear, they propose the possibility of distinct initial inflammatory responses to SARS-CoV-2 (with an initial predisposition 
towards a Th2 response in the eosinophil recovery group); or maybe different inflammatory evolutions, with an immune recovery associated with modification from an initial Th1 inflammatory response to a Th2 response.

Some clinical variables seem to be strongly associated with the odds of requiring readmission, such as fever (temperature over $38{ }^{\circ} \mathrm{C}$ ) at the time of evaluation in the emergency ward. The fact that no patient discharged being on their 10th day of disease progression or later required readmission is noteworthy. This coincides with what has been described about the COVID-19 pathogenesis [8]. Once the first week has been overcome, patients might enter an inflammatory phase with progressive worsening, (in which case they would meet admission criteria) or start recovering until the fever and the respiratory symptoms are resolved.

In our cohort, the D-dimer levels were not correlated with the evolution of the patients, although no patient had a D-dimer level above $1,000 \mathrm{ng} / \mathrm{mL}$ (being an exclusion criterion). The association of D-dimer and severity is well described in the literature $[9,10]$, but its association with respiratory distress and/or mortality significantly increases with values above said threshold. Therefore, it is possible that its differentiating role is much less relevant for values below $1,000 \mathrm{ng} / \mathrm{mL}$.

\section{Limitations of this study}

This study has some limitations, including having been performed only in one hospital, which may hinder its external validity. Older or more comorbid populations might account for a lower proportion of ambulatorily manageable patients, although some select patients might benefit from it. Another relevant issue is the fact that the study does not include information about the cases that were admitted into the hospital upon their first visit, so we do not know if there may have been patients that met discharge criteria but were indeed admitted as inpatients and then eventually required ICU admission or died. However, as a consequence of the great pressure on the emergency ward of our hospital during the pandemic, the ambulatory follow-up program included 133 more patients than the ones analyzed, who did fulfill major admission criteria but were discharged for being clinically stable. Those patients have not been included in the protocol analysis, as the purpose of this paper is to evaluate the performance of the selection criteria, but they somehow suggest that the trend at that time was prioritizing outpatient management whenever possible. It shall be noted that discharged patients with major admission criteria evolved well in general: only $24(18 \%)$ were admitted and 2 of them (1.5\%) eventually died.

Third, the results of the RT-PCR for SARS-CoV-2 is only available in $44 \%$ of the cases, and a positive result is only present in $30 \%$ of the cohort. We consider this to be a consequence of two factors. The protocols from the valid health authority at each moment have changed widely during the pandemic. During most of the study period, performing the test was only authorized for patients admitted into hospitalization or in dubious cases, and hence the diagnosis of typical cases was based on clinical, radiological and laboratory findings. Besides, $29 \%$ of the patients on whom PCR was performed have a negative result. We do not believe this fact to impair the reliability of our results, since PCR sensitivity in our institution was found to be $65-70 \%$, only PCRnegative patients with high clinical suspicion were included in the study, and positive predictive value for COVID-19 diagnosis in Madrid, during the peak of the pandemic was remarkably high. In addition, there were more tested patients among those who were readmitted. Chances of being tested were higher for them, as they were finally admitted into the hospital for clinical deterioration.

Finally, we have detected a great difference between groups regarding the prescribed treatment. This might be a problem in the setting of highly effective treatments, but the evidence available at the moment does not suggest that beta-lactams or azithromycin have a relevant therapeutic role in COVID-19 pneumonia [11-13].

For all these limitations, it would be convenient to carry out further studies, larger and multicentric, in other populations and/or epidemiological situations. This would allow not only to grant greater external validity to our results but also to optimize our selection criteria, such as including information derived from our multivariate analysis.

\section{Conclusions}

Our study shows that ambulatory management of patients with low-risk COVID-19 pneumonia can be safe, whenever they are adequately selected. The management protocol presented in this study has allowed us to avoid $30 \%$ of the admissions for COVID-19 pneumonia in our hospital, with a very low readmission rate and no mortality. Hypertension, high fever at the time of evaluation, leukopenia and increased LDH were associated with a higher risk for readmission, while a high eosinophil count and a longer time since the start of symptoms were associated with low risk. Nevertheless, more studies would be helpful to confirm our findings.

Author contributions We thank JCR, JMAS, CBM, METV, RCA, $\mathrm{MABC}, \mathrm{EZM}$ and all the colleagues from the Emergency Ward of the Infanta Cristina Hospital for the huge effort done during the COVID19 pandemic. We thank also SAP Spain for the donation of the pulse oximeters.

Funding The authors received no specific funding for this work. 


\section{Compliance with ethical standards}

Conflict of interest The author declare that they have no competing interest.

Ethical approval The Clinical Research Ethics Committee of Puerta de Hierro Universitary Hospital considers that an opinion or follow-up by the Committee is not necessary as this is a biomedical research project and that there is no ethical or legal impediment for its realization.

Informed consent For this type of study, no informed consent is required.

\section{References}

1. Ministry of Health (2020) Actualización $n^{\circ} 122$. Enfermedad por el coronavirus (COVID-19). https://www.mscbs.gob.es/en/profe sionales/saludPublica/ccayes/alertasActual/nCov-China/docum entos/Actualizacion_122_COVID-19.pdf. Accessed 3 Jun 2020

2. Zhou F, Yu T, Du R et al (2020) Clinical course and risk factors for mortality of adult inpatients with COVID-19 in Wuhan, China: a retrospective cohort study. Lancet 395(10229):10541062 ([published correction appears in Lancet. 2020 Mar 28;395(10229):1038])

3. Wu C, Chen X, Cai Y et al (2020) Risk factors associated with acute respiratory distress syndrome and death in patients with coronavirus disease 2019 pneumonia in Wuhan, China. JAMA Intern Med 180(7):934-943

4. Fine MJ, Auble TE, Yealy DM et al (1997) A prediction rule to identify low-risk patients with community-acquired pneumonia. N Engl J Med 336(4):243-250

5. Liu F, Xu A, Zhang Y et al (2020) Patients of COVID-19 may benefit from sustained Lopinavir-combined regimen and the increase of Eosinophil may predict the outcome of COVID-19 progression. Int J Infect Dis 95:183-191

6. Sun S, Cai X, Wang H et al (2020) Abnormalities of peripheral blood system in patients with COVID-19 in Wenzhou, China. Clin Chim Acta 507:174-180

7. Mateos M, Sierra E, Casado I et al (2021) the prognostic value of eosinophil recovery in covid-19: a multicentre, retrospective cohort study on patients hospitalised in Spanish Hospitals. J Clin Med 10(2):E305

8. Lin L, Lu L, Cao W, Li T (2020) Hypothesis for potential pathogenesis of SARS-CoV-2 infection-a review of immune changes in patients with viral pneumonia. Emerg Microbes Infect 9(1):727-732

9. Zhang L, Yan X, Fan Q et al (2020) D-dimer levels on admission to predict in-hospital mortality in patients with Covid-19. J Thromb Haemost 18(6):1324-1329

10. Li Y, Zhao K, Wei $\mathrm{H}$ et al (2020) Dynamic relationship between D-dimer and COVID-19 severity. Br J Haematol 190(1):e24-e27

11. Molina JM, Delaugerre C, Le Goff J et al (2020) No evidence of rapid antiviral clearance or clinical benefit with the combination of hydroxychloroquine and azithromycin in patients with severe COVID-19 infection. Med Mal Infect 50(4):384

12. Rosenberg ES, Dufort EM, Udo T et al (2020) Association of treatment with hydroxychloroquine or azithromycin with in-hospital mortality in patients with COVID-19 in New York State. JAMA 323(24):2493-2502

13. Gbinigie K, Frie K (2020) Should azithromycin be used to treat COVID-19? A rapid review. BJGP Open. https://doi.org/10.3399/ bjgpopen20X101094

Publisher's Note Springer Nature remains neutral with regard to jurisdictional claims in published maps and institutional affiliations. 